\title{
Razão Neutrófilo-Linfócito e Aterosclerose da Aorta Abdominal entre Indivíduos Assintomáticos
}

\author{
Neutrophil-To-Lymphocyte Ratio and Abdominal Aortic Atherosclerosis among Asymptomatic Individuals \\ Bárbara Said Marin, ${ }^{10}$ Fernando Cesena, ${ }^{10}$ Antonio Gabriele Laurinavicius, ${ }^{1}$ Raul D. Santos, ${ }^{1,2}$ Marcio Sommer \\ Bittencourt ${ }^{2}$ (1) \\ Hospital Israelita Albert Einstein, ${ }^{1}$ São Paulo, SP - Brasil \\ Universidade de São Paulo, ${ }^{2}$ São Paulo, SP - Brasil
}

\section{Resumo}

Fundamento: A razão neutrófilo-linfócito (RNL) tem sido proposta como um marcador inflamatório possivelmente associado a aterosclerose coronariana, embora a maioria dos dados atuais seja restrita à fase aguda. Além disso, a associação entre a RNL e a aterosclerose extracoronariana ainda não está clara.

Objetivo: Analisar a associação entre a RNL e aterosclerose da aorta abdominal (AtAA).

Métodos: Foram incluídos pacientes assintomáticos submetidos a um programa de rastreamento. A AtAA foi avaliada através de ultrassom. Os números absolutos de leucócitos e linfócitos foram utilizados para calcular a RNL. Foi estabelecido um nível de significância estatística de 0,05 .

Resultados: De 36.985 indivíduos (idade: $42 \pm 10$ anos, $72 \%$ homens), foi identificada a presença de AtAA em $7 \%$. Aqueles com AtAA eram mais velhos e tinham maior propensão a serem homens e diabéticos. A presença de AtAA foi associada a RNL aumentada (odds ratio [OR] 1,17; intervalo de confiança de 95\% [IC95\%] 1,13-1,21). No entanto, a associação deixou de ser significativa quando a análise foi ajustada para os fatores de risco (OR 1,02; IC95\% 0,97-1,06), principalmente devido à inclusão da idade no modelo. Quando os neutrófilos e linfócitos foram analisados separadamente, a associação negativa entre os linfócitos e a RNL foi invertida com a inclusão da idade, o que sugere um forte efeito confundidor da idade na relação entre linfócitos e aterosclerose. Por fim, a associação entre os neutrófilos e a AtAA deixou de ser significativa após o ajuste adicional para os fatores de risco tradicionais, mas não apenas para a idade.

Conclusão: Embora a RNL tenha se associado a AtAA, foi principalmente devido ao efeito confundidor da idade. No geral, os resultados sugerem um papel limitado da contagem de leucócitos como biomarcador de AtAA.

Palavras-chave: Aterosclerose; Biomarcadores; Linfócitos, Neutrófilos, Fatores de Risco.

\footnotetext{
Abstract

Background: Neutrophil-to-lymphocyte ratio (NLR) has been proposed as an inflammatory marker that might be associated with coronary atherosclerosis, although most of the current data is restricted to the acute setting. Additionally, the association of NLR with extracoronary
} atherosclerosis and stable disease remains unclear.

Objective: To analyze the association between NLR and abdominal aortic atherosclerosis (AAAt).

Methods: We included asymptomatic individuals who underwent a health screening program. AAAt was measured by ultrasound. Absolute leukocyte and lymphocyte counts were used to calculate the NLR. The level of significance for statistical analysis was 0.05.

Results: Among 36,985 individuals (age: $42 \pm 10$ years, 72\% male), AAAt was identified in 7\%. Those with AAAt were older and more likely to be male and diabetic. Presence of AAAt was associated with increased NLR (odds ratio [OR] 1.17; 95\% confidence interval [Cl] 1.13-1.21). However, this association was no longer significant when the analysis was adjusted for risk factors (OR 1.02; 95\% CI 0.97-1.06), mostly due to the inclusion of age in the model. When neutrophils and lymphocytes were analyzed separately, the negative association between lymphocytes and AAAt was inverted once age was accounted for, suggesting a strong confounding effect of age on the relationship between lymphocytes and atherosclerosis. Finally, the association of neutrophils and AAAt lost significance after an additional adjustment for traditional risk factors, but not age alone.

Conclusion: Although the NLR was associated with AAAt, this was largely due to the confounding effect of age. Overall, the results suggest a limited role of leukocyte measurements as biomarkers of AAAt.

Keywords: Atherosclerosis; Biomarkers; Lymphocytes; Neutrophils; Risk Factors.

Full texts in English - http://www.arquivosonline.com.br

Correspondência: Bárbara Said Marin •

Hospital Israelita Albert Einstein - Av. Prof. Francisco Morato, 4.293. CEP 05521-200, São Paulo, SP - Brasil

E-mail: bsmarin.einstein@gmail.com

Artigo recebido em 01/11/2020, revisado em 23/03/2021, aceito em 12/05/2021

DOI: https://doi.org/10.36660/abc.20201163 


\section{Introdução}

As doenças cardiovasculares são a principal causa de morte no mundo. ${ }^{1}$ A combinação de fatores de risco, como diabetes, hipertensão, dislipidemia, obesidade e tabagismo, pode causar o desenvolvimento de aterosclerose. Nos estágios iniciais da formação de placa de ateroma, lipoproteínas de baixa densidade (low-density lipoproteins, LDLs) circulantes, no contexto da disfunção endotelial, penetram e se acumulam na túnica íntima das artérias. Quando oxidadas, as partículas de LDL podem dar início a uma resposta inflamatória que culmina no recrutamento de monócitos/macrófagos para a região da placa e ativa a imunidade inata e adaptativa. Portanto, o crescimento e as complicações das placas ateroscleróticas são uma resposta inflamatória imunomediada. ${ }^{2}$

Vários estudos demonstraram a relação entre a contagem de glóbulos brancos e o risco de doença arterial coronariana (DAC). ${ }^{3} \mathrm{O}$ estado inflamatório sistêmico leva a um aumento dos neutrófilos, e o estresse agudo provocado por complicações das placas ateroscleróticas leva a uma diminuição dos linfócitos. ${ }^{4-6}$ Os neutrófilos também foram associados a uma chance maior de eventos, ${ }^{7}$ enquanto a taxa de linfócitos foi significativamente menor em pacientes com eventos cardíacos que ainda apresentavam um risco maior de eventos futuros (por exemplo, DAC, angina instável e morte cardíaca). ${ }^{8,9}$ A razão neutrófilo-linfócito ( $\left.R N L\right)$ é um marcador inflamatório que tem sido extensamente estudado nos últimos anos e aparenta ter um papel importante não apenas na predição de eventos cardiovasculares, mas também na predição de desfechos clínicos no contexto de hemorragias cerebrais, ${ }^{10,11}$ eventos cardíacos maiores ${ }^{12} \mathrm{e}$ sepse e doenças infecciosas. ${ }^{13}$ Dessa forma, esse simples índice, derivado de um teste de baixo custo e facilmente reproduzível, pode conter informações relevantes sobre o risco de desfechos cardiovasculares. ${ }^{14}$

A associação entre a RNL e prognóstico em diferentes tipos de doença cardiovascular, como em síndromes coronarianas agudas, arritmias cardíacas, insuficiência cardíaca congestiva descompensada, substituição da valva aórtica transcateter e doenças valvulares cardíacas, ${ }^{15}$ tem sido relatada por diversos autores. No entanto, alguns estudos ainda apresentam limitações nas análises multivariadas, que nem sempre consideram todos os fatores confundidores, o que compromete os resultados da verdadeira associação entre a RNL e o risco cardiovascular. Além disso, não há dados disponíveis que correlacionem a RNL com doença cardiovascular aterosclerótica subclínica e apliquem a RNL à estratificação de risco cardiovascular. Assim, os objetivos deste estudo foram correlacionar a presença de aterosclerose subclínica com a RNL e avaliar se a RNL adiciona discriminação aos fatores de risco tradicionais.

\section{Métodos}

\section{População do estudo}

Foram incluídos todos os indivíduos submetidos a um programa de rastreamento no Centro de Medicina Preventiva do Hospital Israelita Albert Einstein, em São Paulo, Brasil, no período de 2006 a 2015. O programa consiste em avaliações clínica e laboratorial extensas e ultrassom abdominal. O protocolo do estudo foi aprovado pelo Conselho de Revisão Institucional, com dispensa de consentimento informado.

\section{Avaliação clínica e laboratorial}

Os dados demográficos, o histórico médico e o uso de medicamentos foram obtidos através de questionários padrões. O estado de tabagismo foi categorizado em fumante (pelo menos um cigarro nos últimos 30 dias) ou ex-fumante e não fumante. A altura $(\mathrm{m})$ e o peso $(\mathrm{kg})$ foram aferidos com um estadiômetro e uma balança médica antropométrica, respectivamente, para calcular o índice de massa corporal (IMC, $\left.\mathrm{kg} / \mathrm{m}^{2}\right)$. A pressão arterial foi aferida três vezes na posição sentada com um esfigmomanômetro aneroide de acordo com o método padrão recomendado pela American Heart Association. ${ }^{16}$ A hipertensão foi definida como níveis de pressão arterial $\geq 140 / 90 \mathrm{mmHg}$ durante a avaliação ou uso de medicamentos para pressão alta. O diabetes melito foi definido como valores de glicemia de jejum $\geq 5,55 \mathrm{mmol} / \mathrm{L}$ ou uso de tratamento medicamentoso para hiperglicemia. A dislipidemia foi definida como nível elevado de triglicerídeos (TG) ( $\geq 1,7 \mathrm{mmol} / \mathrm{L})$, nível elevado de colesterol LDL $(4,12 \mathrm{mmol} / \mathrm{L})$ e baixo nível de colesterol de lipoproteína de alta densidade (high-density lipoprotein, HDL) $(<1,02 \mathrm{mmol} / \mathrm{L}$ para homens e $<1,28 \mathrm{mmol} / \mathrm{L}$ para mulheres $)$ ou uso de medicamentos hipolipemiantes. Foram coletadas amostras de sangue após o jejum recomendado de 12 horas. As amostras foram processadas no laboratório do Centro de Medicina Preventiva do Hospital Israelita Albert Einstein. Os níveis de colesterol total, TG, colesterol HDL, glicose e células sanguíneas foram determinados através de testes laboratoriais automatizados padrões. ${ }^{17} \mathrm{O}$ colesterol LDL foi determinado diretamente ou pela fórmula de Friedewald ${ }^{18}$ para níveis de TG $\geq 4,5 \mathrm{mmol} / \mathrm{L} \mathrm{e}<4,5 \mathrm{mmol} / \mathrm{L}$, respectivamente.

\section{Ultrassom abdominal}

Foi realizado ultrassom abdominal com abordagem padrão por radiologistas certificados. A aorta abdominal foi sistematicamente avaliada para a presença de aterosclerose. Foi definida como aterosclerose da aorta abdominal (AtAA) a presença de placa lipídica ou de ateroma no relatório do ultrassom abdominal.

\section{Análise estatística}

Variáveis contínuas são apresentadas como médias e desvios padrão ou como medianas e intervalos interquartis, conforme apropriado. A normalidade foi avaliada através de inspeção visual de histogramas. As variáveis categóricas são apresentadas como números absolutos e porcentagens. As diferenças nas características basais dos indivíduos de acordo com os quintis da RNL e a presença de AtAA foram avaliadas por meio do teste $t$ para amostras independentes; da análise de variância (ANOVA) unidirecional para variáveis contínuas com distribuição normal; e do teste da soma de postos de Wilcoxon ou do teste de Kruskal-Wallis 
para variáveis com distribuição não normal. O teste do qui-quadrado foi utilizado para as variáveis categóricas. As associações entre RNL, neutrófilos, linfócitos e AtAA foram primeiramente testadas por análise multivariada e posteriormente ajustadas para sexo e idade com modelos de regressão logística. As análises multivariadas adicionais incluíram idade, sexo, tabagismo, hipertensão arterial, diabetes e dislipidemia. Os testes foram conduzidos com um nível de significância de 5\%. Todas as análises foram realizadas no software Stata, versão 13.0.

\section{Resultados}

A amostra do estudo incluiu 36.985 indivíduos (homens:

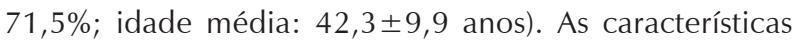
demográficas, clínicas e laboratoriais basais estão apresentadas na Tabela 1 para todos os pacientes e de acordo com os quintis da RNL.

Não houve diferença entre os quintis da RNL para os níveis de colesterol total e colesterol LDL. Os pacientes no quintil mais alto da RNL eram mais velhos e mais propensos a ter diabetes e hipertensão ( $p<0,001$ para todos), tendo apresentado a maior contagem de neutrófilos e a menor contagem de linfócitos ( $p<0,001$ para ambos). Os pacientes no quintil mais baixo da RNL apresentaram o IMC mais baixo $(p=0,027)$, o nível de TG mais baixo $(p<0,001)$ e o nível de colesterol HDL mais alto $(p<0,001)$. Também apresentaram a menor contagem de neutrófilos e a maior contagem de linfócitos ( $p<0,001$ para ambos).
A AtAA foi identificada por ultrassom em $7 \%$ dos pacientes. Em comparação aos participantes sem AtAA, aqueles com AtAA eram mais velhos, mais frequentemente do sexo masculino e fumantes ou ex-fumantes e com maior frequência possuíam um diagnóstico de diabetes, hipertensão ou dislipidemia (Tabela 2).

A RNL foi mais alta em pacientes com AtAA em comparação àqueles sem AtAA. Após a análise multivariada, níveis mais elevados de RNL foram diretamente associados a aterosclerose. Quando analisados separadamente, os neutrófilos foram diretamente associados a AtAA, enquanto os linfócitos foram negativamente associados. No entanto, não houve associação entre a RNL e aterosclerose na análise multivariada quando ajustada para sexo, idade e fatores de risco, principalmente devido à inclusão da idade. A associação negativa entre linfócitos e AtAA foi revertida com a inclusão da idade no modelo, o que sugere a presença de um efeito confundidor. A associação entre neutrófilos e AtAA deixou de ser significativa após o ajuste para os fatores de risco tradicionais, mas não apenas para a idade.

\section{Discussão}

O presente estudo demonstrou que não há associação entre RNL e aterosclerose aórtica após a consideração dos confundidores conhecidos. Apesar da associação significativa observada na análise univariada, esses efeitos parecem estar amplamente relacionados ao efeito confundidor da idade, uma vez que a RNL está fortemente

Tabela 1 - Características basais dos participantes do estudo e comparação entre os quintis da razão neutrófilo-linfócito

\begin{tabular}{|c|c|c|c|c|c|c|c|}
\hline & Total & Quintil 1 & Quintil 2 & Quintil 3 & Quintil 4 & Quintil 5 & p \\
\hline Idade (anos) & $42,3 \pm 9,9$ & $40,3 \pm 9,8$ & $41,6 \pm 9,7$ & $42,4 \pm 9,7$ & $42,8 \pm 9,7$ & $44,4 \pm 10,3$ & $<0,001$ \\
\hline Sexo masculino (\%) & $26.248(71,5 \%)$ & $5.144(70 \%)$ & $5.430(74 \%)$ & $5.365(73 \%)$ & $5.317(72 \%)$ & $4.992(68 \%)$ & $<0,001$ \\
\hline Tabagismo (\%) & & & & & & & 0,042 \\
\hline Ex-fumante & $4.790(13,1 \%)$ & $958(13,1 \%)$ & $963(13,1 \%)$ & $956(13 \%)$ & $965(13,2 \%)$ & $948(12,9 \%)$ & \\
\hline Fumante & $3.759(10,3 \%)$ & $726(9,9 \%)$ & $732(10 \%)$ & $720(9,8 \%)$ & $738(10,1 \%)$ & $843(11,5 \%)$ & \\
\hline Diabetes melito (\%) & $936(2,6 \%)$ & $116(2 \%)$ & $157(2 \%)$ & $178(2 \%)$ & $200(3 \%)$ & $285(4 \%)$ & $<0,001$ \\
\hline Hipertensão (\%) & $4.819(13,1 \%)$ & $782(11 \%)$ & $841(11 \%)$ & $963(13 \%)$ & $999(14 \%)$ & $1.234(17 \%)$ & $<0,001$ \\
\hline IMC $\left(\mathrm{kg} / \mathrm{m}^{2}\right)$ & $26,5 \pm 4,3$ & $26,2 \pm 4,2$ & $26,4 \pm 4,3$ & $26,7 \pm 4,3$ & $26,8 \pm 4,4$ & $26,5 \pm 4,3$ & 0,027 \\
\hline Dislipidemia (\%) & $9.927(27 \%)$ & $1.878(26 \%)$ & $1.990(27 \%)$ & $2.093(28 \%)$ & $1.960(27 \%)$ & $2.006(27 \%)$ & 0,002 \\
\hline Triglicerídeos* $(\mathrm{mg} / \mathrm{dL})$ & $112(79-161)$ & $107(77-156)$ & $113(79-163)$ & $112(81-162)$ & $115(80-163)$ & $112(79-158)$ & $<0,001$ \\
\hline Colesterol (mg/dL) & $196,9 \pm 37,6$ & $198,2 \pm 37,7$ & $199 \pm 37,3$ & $197,7 \pm 38$ & $196,8 \pm 37,5$ & $193 \pm 37,2$ & 0,328 \\
\hline Colesterol HDL (mg/dL) & $49,1 \pm 13,6$ & $50,6 \pm 14,7$ & $49,1 \pm 13,5$ & $48,7 \pm 13,3$ & $48,2 \pm 13$ & $49 \pm 13,6$ & $<0,001$ \\
\hline Colesterol LDL (mg/dL) & $121,7 \pm 34$ & $121,9 \pm 34,3$ & $123,1 \pm 33,7$ & $122,8 \pm 34,2$ & $122,1 \pm 34$ & $118,6 \pm 33,4$ & 0,122 \\
\hline Leucócitos $\left(/ \mathrm{mm}^{3}\right)$ & $6.472 \pm 1.575$ & $5.918 \pm 1.368$ & $6.202 \pm 1.364$ & $6.344 \pm 1.397$ & $6.609 \pm 1.502$ & $7.286 \pm 1.840$ & $<0,001$ \\
\hline Neutrófilos $\left(/ \mathrm{mm}^{3}\right)$ & $3.600 \pm 1.195$ & $2.616 \pm 680$ & $3.166 \pm 718$ & $3.494 \pm 789$ & $3.906 \pm 918$ & $4.818 \pm 1.399$ & $<0,001$ \\
\hline Linfócitos $\left(/ \mathrm{mm}^{3}\right)$ & $2.117 \pm 580$ & $2.554 \pm 615$ & $2.285 \pm 515$ & $2.101 \pm 473$ & $1.949 \pm 452$ & $1.696 \pm 430$ & $<0,001$ \\
\hline Proteína C reativa* & $0,12(0,06-0,27)$ & $0,10(0,05-0,22)$ & $0,11(0,06-0,23)$ & $0,12(0,06-0,26)$ & $0,13(0,07-0,28)$ & $0,17(0,08-0,38)$ & $<0,001$ \\
\hline
\end{tabular}


Tabela 2 - Características dos pacientes de acordo com a presença de aterosclerose no ultrassom abdominal

\begin{tabular}{|c|c|c|c|}
\hline & Aterosclerose & Sem aterosclerose & $p$ \\
\hline Idade (anos) & $57,2 \pm 8,3$ & $41,2 \pm 9,1$ & $<0,001$ \\
\hline Sexo masculino (\%) & $2.132(82 \%)$ & $24.476(71 \%)$ & $<0,001$ \\
\hline Tabagismo (\%) & & & $<0,001$ \\
\hline Ex-fumante & $810(31,2 \%)$ & $4.042(11,7 \%)$ & \\
\hline Fumante & $379(14,6 \%)$ & $3.444(10 \%)$ & \\
\hline Diabetes melito (\%) & $253(10 \%)$ & $700(2 \%)$ & $<0,001$ \\
\hline Hipertensão (\%) & $1.004(39 \%)$ & $3.896(11 \%)$ & $<0,001$ \\
\hline IMC $\left(\mathrm{kg} / \mathrm{m}^{2}\right)$ & $27,3 \pm 3,8$ & $26,4 \pm 4,3$ & $<0,001$ \\
\hline Dislipidemia (\%) & $1.419(55 \%)$ & $8.655(25 \%)$ & $<0,001$ \\
\hline Triglicerídeos* (mg/dL) & $128(91-178)$ & $110(78-159)$ & $<0,001$ \\
\hline Colesterol (mg/dL) & $196,3 \pm 42$ & $196,9 \pm 37,3$ & 0,21 \\
\hline Colesterol HDL (mg/dL) & $46,5 \pm 12,7$ & $49,2 \pm 13,7$ & $<0,001$ \\
\hline Colesterol LDL (mg/dL) & $120,7 \pm 37,8$ & $121,7 \pm 33,7$ & 0,07 \\
\hline Leucócitos $\left(/ \mathrm{mm}^{3}\right)$ & $6.611,7 \pm 1.775,5$ & $6.474,8 \pm 1.665,2$ & $<0,001$ \\
\hline Neutrófilos $\left(/ \mathrm{mm}^{3}\right)$ & $3.732 \pm 1.248,6$ & $3.590,7 \pm 1.191,1$ & $<0,001$ \\
\hline Linfócitos $\left(/ \mathrm{mm}^{3}\right)$ & $2.077,8 \pm 822,5$ & $2.126,6 \pm 592,9$ & $<0,001$ \\
\hline
\end{tabular}

*Mediana (intervalo interquartil). IMC: índice de massa corporal; HDL: lipoproteína de alta densidade; LDL: lipoproteína de baixa densidade; Observação: 0 teste $t$ foi utilizado para variáveis contínuas. 0 teste de Mann-Whitney foi utilizado para os triglicerídeos. 0 teste do qui-quadrado foi utilizado para as variáveis categóricas.

correlacionada à idade nesta população. Coletivamente, este estudo sugere que a RNL não serve como um marcador de aterosclerose em pacientes assintomáticos que participaram de um programa de rastreamento.

Já se sabe que os biomarcadores de inflamação estão associados a um risco maior de eventos cardiovasculares e que algumas terapias anti-inflamatórias são capazes de preveni-los. ${ }^{19} \mathrm{~A}$ identificação de pacientes de alto riso é fundamental para explorar o tratamento ideal, sendo que a RNL pode ser um importante biomarcador a ser identificado nesses pacientes, a qual está associada ao prognóstico em doenças ateroscleróticas, bem como em sua prevalência, como aponta a Figura 1.

A associação entre a RNL como um preditor de mortalidade e desfechos coronarianos agudos foi demonstrada por vários estudos. Em doenças agudas, os resultados estão associados a níveis elevados de neutrófilos, ${ }^{12}$ os mediadores de respostas a lesões miocárdicas como infarto do miocárdio. Esse resultado também foi demonstrado em estudos sobre doença coronariana estável. A contagem de linfócitos relativa está associada à sobrevida de pacientes com DAC, ${ }^{9}$ enquanto outros biomarcadores, como proteína $\mathrm{C}$ reativa (PCR) e leucócitos, estão associados a desfechos crônicos e agudos. ${ }^{20} \mathrm{~A}$ PCR, como a RNL, é um biomarcador associado a inflamação e predição de risco de mortalidade. Em modelos de estudo com apenas RNL ou PCR, cada parâmetro isolado foi capaz de predizer o risco. No entanto, quando ambos os biomarcadores foram aplicados, houve uma melhora significativa na predição. ${ }^{21} \mathrm{Em}$ contrapartida, os resultados deste estudo não corroboram esses achados. Embora os resultados de outros estudos afirmem que a RNL seja um preditor independente de mortalidade cardiovascular, a presente análise demonstrou que há um forte fator confundidor relacionado à inclusão da idade no modelo.

Como a RNL se correlaciona à idade do paciente, a análise deve ser ajustada de acordo. No entanto, até o momento, não encontramos nenhum estudo que tenha realizado tal ajuste. Todas as análises são baseadas em fatores de risco e prognóstico. Como a idade é um ponto importante de comparação entre os pacientes, o ajuste é extremamente necessário.

Há diferenças entre a população do presente estudo e de outros estudos. Foi analisado um grupo mais jovem de uma grande população, o qual possuía boas condições socioeconômicas e consistia principalmente em homens e pessoas brancas. A maioria dos estudos incluem populações do hemisfério Norte, enquanto o presente estudo incluiu uma população de um país tropical da América Latina. Além disso, foram realizados testes laboratoriais e avaliação sistemática dos fatores de risco. Na análise estatística, foram realizados ajustes detalhados para os fatores confundidores. A RNL, os neutrófilos e os linfócitos foram analisados separadamente (Tabela 3).

O presente estudo deve, no entanto, ser compreendido no contexto do seu desenho. Devido ao caráter transversal dos dados, não foi possível inferir causalidade. A população selecionada incluiu uma prevalência maior de homens, em sua maioria jovens, o que leva a uma baixa prevalência de doença e pode atenuar a capacidade de identificar associações. Além disso, o foco do presente estudo foi a avaliação de aterosclerose aórtica, que não necessariamente 


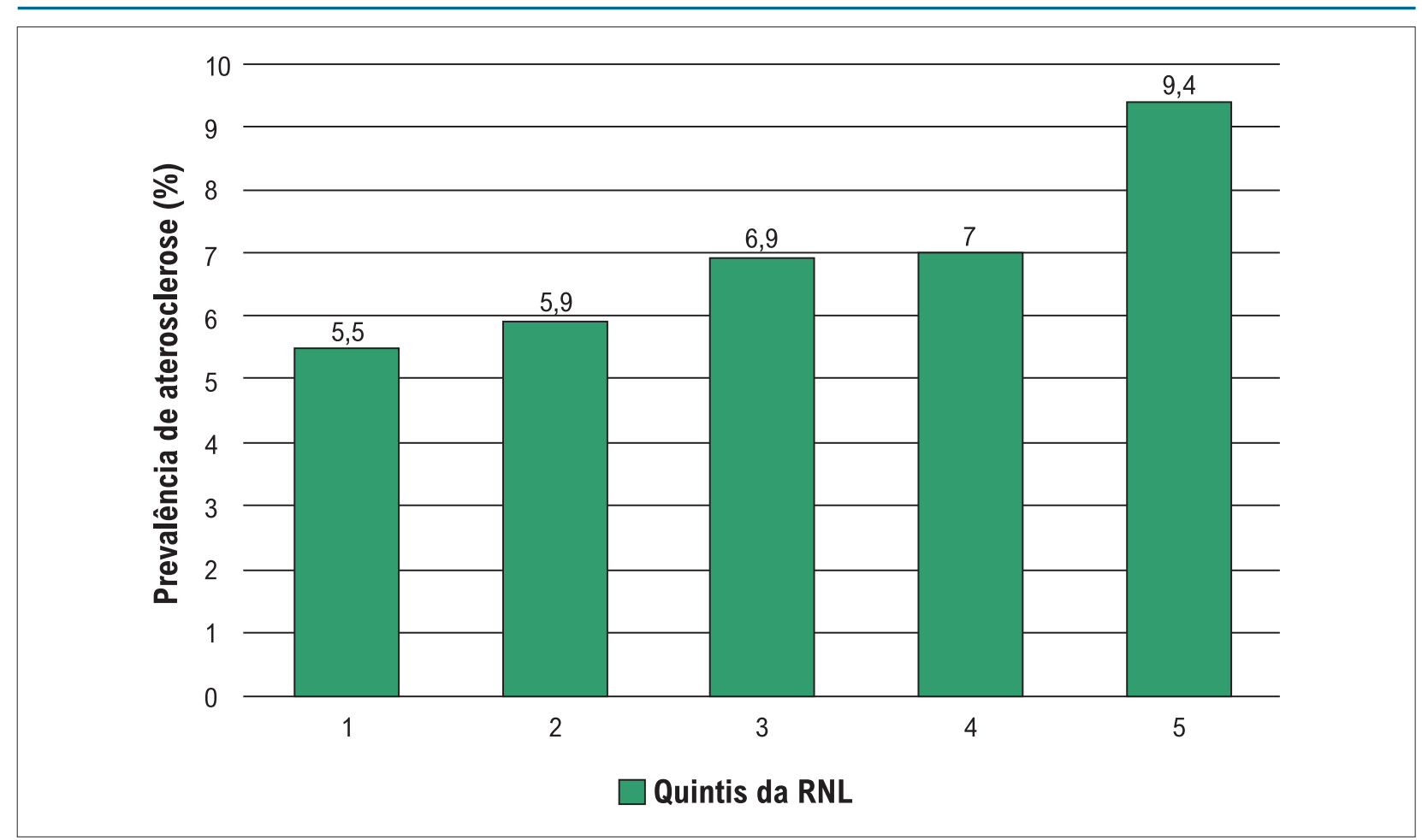

Figura 1 - Prevalência de aterosclerose de acordo com os quintis da razão neutrófilo-linfócito (RNL) $(p<0,001)$.

Tabela 3 - Análise multivariada da relação entre a razão neutrófilo-linfócito, neutrófilos ou linfócitos e aterosclerose abdominal

\begin{tabular}{lccc}
\hline \multicolumn{4}{c}{ Odds ratio para aterosclerose (intervalo de confiança de 95\%) } \\
\hline RNL & Não ajustado & Modelo 1 & Modelo 2 \\
\hline Neutrófilo & $1,17(1,13-1,21)$ & $1,00(0,96-1,05)$ & $1,00(0,95-1,04)$ \\
\hline Linfócito & $1,07(1,03-1,11)$ & $1,05(1,01-1,10)$ & $0,99(0,95-1,04)$ \\
\hline
\end{tabular}

RNL: razão neutrófilo-linfócito. Modelo 1: Ajustado para idade e sexo. Modelo 2: Ajustado para idade, sexo, tabagismo, hipertensão, diabetes e dislipidemia.

possui o mesmo processo fisiopatológico da aterosclerose em outros locais, como a artéria coronária.

\section{Conclusão}

Embora tenha havido associação entre aterosclerose e RNL, foi principalmente devido ao efeito confundidor da idade. A associação entre neutrófilos e linfócitos e aterosclerose deixou de ser significativa ao serem incluídos em modelos multivariados. Os resultados sugerem um papel limitado do biomarcador na avaliação de aterosclerose subclínica.

\section{Contribuição dos autores}

Concepção e desenho da pesquisa: Cesena F, Laurinavicius AG, Santos RD, Bittencourt MS; Obtenção de dados: Bittencourt MS; Análise e interpretação dos dados e Revisão crítica do manuscrito quanto ao conteúdo intelectual importante: Marin BS, Cesena F, Laurinavicius AG, Santos RD, Bittencourt MS; Análise estatística e
Redação do manuscrito: Marin BS, Bittencourt MS.

\section{Potencial conflito de interesse}

Não há conflito com o presente artigo

\section{Fontes de financiamento}

O presente estudo não teve fontes de financiamento externas.

\section{Vinculação acadêmica}

Não há vinculação deste estudo a programas de pósgraduação.

\section{Aprovação ética e consentimento informado}

Este artigo não contém estudos com humanos ou animais realizados por nenhum dos autores. 


\section{Referências}

1. World Health Organization. Cardiovascular Diseases (CVDs) Fact Sheet. Geneva: World Health Organization; 2017.

2. Gisterå A, Hansson GK. The Immunology of Atherosclerosis. Nat Rev Nephrol. 2017;13(6):368-80. doi: 10.1038/nrneph.2017.51.

3. Horne BD, Anderson JL, John JM, Weaver A, Bair TL, Jensen KR, et al. Which White Blood Cell Subtypes Predict Increased Cardiovascular Risk? J Am Coll Cardiol. 2005;45(10):1638-43. doi: 10.1016/j.jacc.2005.02.054.

4. Zazula AD, Précoma-Neto D, Gomes AM, Kruklis H, Barbieri GF, Forte RY, et al. An Assessment of Neutrophils/Lymphocytes Ratio in Patients Suspected of Acute Coronary Syndrome. Arq Bras Cardiol. 2008;90(1):31-6. doi: 10.1590/s0066-782×2008000100006.

5. Menon V, Lessard D, Yarzebski J, Furman MI, Gore JM, Goldberg RJ. Leukocytosis and Adverse Hospital Outcomes After Acute Myocardial Infarction. Am J Cardiol. 2003;92(4):368-72. doi: 10.1016/s0002 9149(03)00651-9.

6. Thomson SP, McMahon LJ, Nugent CA. Endogenous Cortisol: A Regulator of the Number of Lymphocytes in Peripheral Blood. Clin Immunol Immunopathol. 1980;17(4):506-14. doi: 10.1016/0090-1229(80)90146-4.

7. Kawaguchi H, Mori T, Kawano T, Kono S, Sasaki J, Arakawa K. Band Neutrophil Count and the Presence and Severity of Coronary Atherosclerosis. Am Heart J. 1996;132(1Pt 1):9-12. doi: 10.1016/s0002-8703(96)90384-1.

8. Zouridakis EG, Garcia-Moll X, Kaski JC. Usefulness of the Blood Lymphocyte Count in Predicting Recurrent Instability and Death in Patients with Unstable Angina Pectoris. Am J Cardiol. 2000;86(4):449-51. doi: 10.1016/s00029149(00)00963-2.

9. Ommen SR, Gibbons RJ, Hodge DO, Thomson SP. Usefulness of the Lymphocyte Concentration as a Prognostic Marker in Coronary Artery Disease. Am J Cardiol. 1997;79(6):812-4. doi: 10.1016/s00029149(96)00878-8.

10. Lattanzi S, Brigo F, Trinka E, Cagnetti C, Di Napoli M, Silvestrini M. Neutrophil-to-Lymphocyte Ratio in Acute Cerebral Hemorrhage: A System Review. Transl Stroke Res. 2019;10(2):137-45. doi: 10.1007/s12975-0180649-4.

11. Lattanzi S, Cagnetti C, Rinaldi C, Angelocola S, Provinciali L, Silvestrini M Neutrophil-to-Lymphocyte Ratio Improves Outcome Prediction of Acute Intracerebral Hemorrhage. J Neurol Sci. 2018;387:98-102. doi: 10.1016/j. jns.2018.01.038.
12. Tamhane UU, Aneja S, Montgomery D, Rogers EK, Eagle KA, Gurm HS. Association Between Admission Neutrophil to Lymphocyte Ratio and Outcomes in Patients with Acute Coronary Syndrome. Am J Cardiol. 2008;102(6):653-7. doi: 10.1016/j.amjcard.2008.05.006

13. Jager CP, van Wijk PT, Mathoera RB, de Jongh-Leuvenink J, van der Poll $\mathrm{T}_{\text {, }}$ Wever PC. Lymphocytopenia and Neutrophil-Lymphocyte Count Ratio Predict Bacteremia Better than Conventional Infection Markers in an Emergency Care Unit. Crit Care. 2010;14(5):192. doi: 10.1186/cc9309.

14. Zazula AD, Précoma-Neto D, Gomes AM, Kruklis H, Barbieri GF, Forte RY, et al. Avaliação da Relação Neutrófilos/Linfócitos em Pacientes com Suspeita de Síndrome Coronariana Aguda. Arq. Bras. Cardiol. 2008;90(1):31-6. doi: 10.1590/S0066-782X2008000100006.

15. Afari ME, Bhat T. Neutrophil to Lymphocyte Ratio (NLR) and Cardiovascular Diseases: An Update. Expert Rev Cardiovasc Ther. 2016;14(5):573-7. doi: 10.1586/14779072.2016.1154788.

16. Perloff D, Grim C, Flack J, Frohlich ED, Hill M, McDonald M, et al. Human Blood Pressure Determination by Sphygmomanometry. Circulation. 1993;88(5Pt1):2460-70. doi: 10.1161/01.cir.88.5.2460.

17. Orthoclinical Diagnostics. Vitros 5600 [Internet]. Markham: Orthoclinica Diagnostics; c2021 [cited 2021 Jul 27]. Available from: https://www. orthoclinicaldiagnostics.com/en-ca/home/vitros-5600

18. Friedewald WT, Levy RI, Fredrickson DS. Estimation of the Concentration of Low-density Lipoprotein Cholesterol in Plasma, without use of the Preparative Ultracentrifuge. Clin Chem. 1972;18(6):499-502.

19. Ridker PM, Everett BM, Thuren T, MacFadyen JG, Chang WH, Ballantyne C, et al. Antiinflammatory Therapy with Canakinumab for Atherosclerotic Disease. N Engl J Med. 2017;377(12):1119-31. doi: 10.1056/ NEJMoa1707914

20. Papa A, Emdin M, Passino C, Michelassi C, Battaglia D, Cocci F. Predictive Value of Elevated Neutrophil-Lymphocyte Ratio on Cardiac Mortality in Patients with Stable Coronary Artery Disease. Clin Chim Acta. 2008;395(12):27-31. doi: 10.1016/j.cca.2008.04.019.

21. Shin HC, Jang JS, Jin HY, Seo JS, Yang TH, Kim DK, et al. Combined Use of Neutrophil to Lymphocyte Ratio and C-Reactive Protein Level to Predict Clinical Outcomes in Acute Myocardial Infarction Patients Undergoing Percutaneous Coronary Intervention. Korean Circ J. 2017;47(3):383-91. doi: 10.4070/kcj.2016.0327. 